\title{
Nonequilibrium relaxation and pattern formation
}

\author{
Christian Maes ${ }^{1}$ and Karel Netočnýn ${ }^{2, \text { f }}$ \\ ${ }^{1}$ Instituut voor Theoretische Fysica, KU Leuven, Belgium \\ ${ }^{2}$ Institute of Physics, Academy of Sciences of the Czech Republic, Prague, Czech Republic
}

We consider a model of nonequilibrium pattern formation. Probes on a ring are in contact with a driven diffusive dynamics and, close to the equidistant probe configuration, the induced forces generate a linear dynamics with nonsymmetric coupling constants. A compound Poisson process describes the nonvariational relaxation to that crystal pattern.

Dedicated to the memory of Roland Dobrushin.

\section{INTRODUCTION}

A mathematical theory that unifies the nature of nonequilibrium phenomena is still under construction. One of the missing links is to systematically describe the forces induced by nonequilibrium baths on tagged particles (= probes) which are coupled to them. The coupling is via an interaction potential between the many bath particles and the (fewer, slower) probes. In thermal equilibrium, i.e., under a reversible bath dynamics with static probes, such forces are derived from thermodynamic potentials (free energies) which also govern the large deviations of the corresponding statistical mechanical system. No such thermodynamic formalism exists in nonequilibrium, at least not comparable in power with the Gibbs equilibrium formalism as established rigorously by the pioneers of mathematical statistical mechanics very much including Roland Dobrushin. We are still learning today how to describe forces induced by "ideal" nonequilibrium baths.

In recent papers [1, 3, 7] we have considered the case of probes interacting with a nonequilibrium density field. We have calculated the induced forces on them and we have demonstrated the emergence of new stable dynamical attractors due to nonequilibrium. In

*Electronic address: netocny@fzu.cz 
the next Section we review the results for probes suspended in a nonequilibrium medium on a ring. In that case a crystal pattern, where the probes are equidistant, becomes a stable fixed point (or, more precisely, a limit circle). A relevant next question is to discuss the corresponding linearized probe-dynamics around that pattern. That is interesting as a physically realized nonvariational flow. Such a relaxation to a nonequilibrium condition is much less studied than the corresponding relaxation to thermal equilibrium. Here, the return to the crystal pattern is not following gradient flow because of an asymmetry in the effective spring constants. We will obtain a full discussion of regimes and time-scales using a compound Poisson process.

The next section gives a more precise description of the model, a driven diffusion on the ring coupled to probes. Its mathematical features are quite simple and an exact solution for the stationary density $\rho_{X}$ was already obtained before. We describe the induced forces. The equidistant configuration of the probes along the ring becomes stable in nonequilibrium. We call it the emerging crystal pattern. The last Section presents a probabilistic argument for its dynamical stability, studying a nonvariational relaxation of the probe positions. Non-reactive interactions substantially modify the way the perturbed probes return to the crystal pattern. Far from equilibrium the relaxation can be mapped exactly onto a compound Poisson process: Any local initial perturbation travels in the form of a Poisson wave with a constant speed in the negative direction (this way creating an "echo" effect) and spreads in a diffusive manner, always keeping the zero mode fixed. The analysis in that Section shows a large time separation between the (fast) echo and the (slow) diffusion. There we approach the spirit of 'statistical mechanics as a branch of probability theory,' in which we cherish the memory of Roland Dobrushin [9]. Dobrushin has made several keycontributions to nonequilibrium statistical mechanics, including the pioneering works [2, 4]. We hope he would have liked to see how new challenges in nonequilibrium require new ideas in probability.

\section{MODEL AND INDUCED FORCES}

The present section is a review of results to prepare the main finding under Section III. Let $\eta \in S^{L}=[0, L]$ be on the ring of size $L$ and write $X=\left(x_{\alpha}\right)_{\alpha=1}^{N}$ for the vector of all 
probe positions on that same ring. We consider the unique smooth solution $\rho_{X}(\eta)>0$ to the stationary Smoluchowski equation (with temperature set to unity),

$$
\partial_{\eta}\left(\left[\partial_{\eta} \Phi(X, \eta)-\varepsilon\right] \rho_{X}(\eta)\right)+\partial_{\eta \eta}^{2} \rho_{X}(\eta)=0 \quad(\eta \bmod L)
$$

where $\varepsilon \geq 0$ is a constant. The total potential is $\Phi(X, \eta)=\sum_{\alpha} u\left(x_{\alpha}-\eta\right)$ with smooth and local interaction $u$ satisfying $u(z)=0$ whenever $|z|>\delta$ for some $\delta<L / 2$, and $u(-z)=u(z)$. We put $\oint \rho_{X}(\eta) \mathrm{d} \eta=: \rho^{o} L$ as the total mass. There is a stationary current

$$
j_{X}=\rho_{X}(\eta)\left[\varepsilon-\sum_{\alpha} u^{\prime}\left(x_{\alpha}-\eta\right)\right]-\partial_{\eta} \rho_{X}(\eta)
$$

which is constant in $\eta$ (from (1)) but depends on the probe positions $x_{\alpha}$, kept fixed in (1). The stationary density $\rho_{X}(\eta)$ can be obtained by direct integration; see e.g. [7, 8].

We focus on the globally strong driving regime, $L \gg \varepsilon^{-1}$. Another length scale is the inverse density $L / N$ of probes. With the range $\delta$ of the interaction, we take that $L / N>2 \delta$ and we assume that the probes are mutually separated

$$
\left|x_{\alpha}-x_{\gamma}\right| \geq 2 \delta \quad \text { for all pairs }(\alpha, \gamma)
$$

which implies that under reversibility, $\varepsilon=0$, the mean forces on them will be zero. By the latter we refer to the induced force on the $\alpha$-probe, which in the quasistatic limit is represented by the mean force

$$
f_{\alpha}(X)=-\int_{0}^{L} \frac{\partial \Phi(X, \eta)}{\partial x_{\alpha}} \rho_{X}(\eta) \mathrm{d} \eta=-\int_{0}^{L} u^{\prime}\left(x_{\alpha}-\eta\right) \rho_{X}(\eta) \mathrm{d} \eta
$$

Observe that the sum of all forces equals

$$
\sum_{\alpha} f_{\alpha}(X)=-\sum_{\alpha} \oint \frac{\partial \Phi(X, \eta)}{\partial x_{\alpha}} \rho_{X}(\eta) \mathrm{d} \eta=\oint \partial_{\eta} \Phi(X, \eta) \rho_{X}(\eta) \mathrm{d} \eta=\left(\varepsilon \rho^{o}-j_{X}\right) L
$$

as follows by integrating the Smoluchowski equation (1) over the ring.

Next we consider the equidistant probe configuration $X^{*}$, with probe positions

$$
x_{\alpha}^{*}=q+\frac{L}{N} \alpha \quad(\alpha \bmod N)
$$

for reference position $q \in S^{L}$ which is arbitrary for the moment. For short, we call that configuration the crystal pattern. That crystal is a quasi-fixed point in the sense that by 
translation symmetry all forces on each probe are clearly identical; from (5) $f_{\alpha}\left(X^{*}\right)=$ $\left(\varepsilon \rho^{o}-j_{X^{*}}\right)=: F^{*}$. In the case of reversibility, $\varepsilon=0$ and $j_{X^{*}}=0$, which yields $F^{*}=0$ and the fixed point $X^{*}$ is trivially marginally stable. (When including also fluctuations induced by the bath then the pattern diffuses away.)

In the nonequilibrium case $(\varepsilon>0)$ the forces are non-zero and, upon linearization,

$$
f_{\alpha}\left(X^{*}+Y\right)=F^{*}+\sum_{\gamma} M_{\alpha \gamma} y_{\gamma}+\ldots
$$

with the stiffness matrix $M_{\alpha \gamma}=\partial f_{\alpha}\left(X^{*}\right) / \partial x_{\gamma}$ taking the form, by using the translation symmetry and the equidistant property of $X^{*}$,

$$
M_{\alpha \gamma}=\left\{\begin{array}{ll}
m_{\gamma-\alpha} & \text { for } \alpha \neq \gamma \\
-\sum_{\gamma \neq 0} m_{\gamma} & \text { for } \alpha \neq \gamma
\end{array} \quad(\alpha, \gamma \bmod N)\right.
$$

Here $\left(m_{\alpha}\right)_{\alpha=1}^{N-1}$ are effective "spring constants" and they are given by the formula (see [7] for derivation)

$$
m_{\alpha}=\frac{B \varepsilon^{2} j_{X^{*}}}{1-e^{-\varepsilon L}} e^{-\frac{\varepsilon L}{N} \alpha}
$$

where $B$ is a form factor dependent on the interaction between probes and particles; explicitly,

$$
B=\int_{-\delta}^{\delta}\left[1-e^{u(z)}\right] e^{\varepsilon z} \mathrm{~d} z \int_{-\delta}^{\delta}\left[e^{-u(z)}-1\right] e^{\varepsilon z} \mathrm{~d} z
$$

We note that all spring constants $m_{\alpha}$ have equal sign which is entirely determined by the sign of $B$. Their novel feature is that they are asymmetric, $m_{N-\alpha} \neq m_{\alpha}$; instead they obey the generalized symmetry relation $m_{N-\alpha}(\epsilon)=m_{\alpha}(-\epsilon)$.

\section{NONEQUILIBRIUM RELAXATION}

As an effective model of the quasistatic motion of the probes around the crystal pattern we consider the overdamped dynamical system $\Gamma \dot{x}_{\alpha}=f_{\alpha}(X), \alpha=1, \ldots, N$, with the parameter $\Gamma>0$ representing friction. We restrict ourselves to the linear regime, $X(t)=X^{*}(t)+Y(t)$ around the crystal pattern $x_{\alpha}^{*}(t)=q^{*}(t)+\alpha L / N$ forming the stationary cycle with $q^{*}(t)=$ $q^{*}(0)+F^{*} t / \Gamma$. Then the linear dynamics of (small) deviations $Y=\left(y_{\alpha}\right)$ has the form, cf. (7),

$$
\Gamma \dot{y}_{\alpha}=\sum_{\gamma} M_{\alpha \gamma} y_{\gamma}=\sum_{\gamma=1}^{N-1} m_{\gamma}\left(y_{\alpha+\gamma}-y_{\alpha}\right)
$$


Before analyzing its properties we remark that this equation can in principle be derived from the dynamics of the entire particle-probe system. Here we skip such a derivation and we simply postulate that dynamics, also neglecting the bath-induced fluctuations. We entirely focus on the consequences of the nonequilibrium-induced asymmetry of the spring constants $m_{\gamma}$ which yield a non-gradient flow.

First we consider the Lyapunov stability of the dynamics (11). Since the zero mode $Y_{0}:=\sum_{\alpha} y_{\alpha}$ is conserved, we restrict on the invariant hypersurface $Y_{0}=0$ and we check that the configuration $y_{\alpha} \equiv 0$ is stable for $B>0$ and unstable for $B<0$, respectively. Indeed, it suffices to check the Lyapunov property of the function $\Lambda(Y)=\sum_{\alpha} y_{\alpha}^{2} / \Gamma$. From (11),

$$
\dot{\Lambda}(Y)=\sum_{\gamma=1}^{N-1} m_{\gamma} \sum_{\alpha=0}^{N-1}\left(y_{\alpha+\gamma}-y_{\alpha}\right)^{2}
$$

which is negative iff $B>0$ as verified from (9). That is equivalent to the positivity of the symmetric part of the stiffness matrix $M+M^{T} \geq 0$, which is a sufficient condition for stability; see e.g. [6]. The condition $B>0$ is immediately satisfied, as is clear from (10), when $u(z)$ does not change sign. Note, however, that $B$ can become negative for interactions which are both attractive and repulsive, depending on distance.

Second, for a fixed $B>0$, we want to understand how an initial perturbation relaxes to the equidistant stationary configuration, i.e., what is the relaxation profile solving the linear evolution (11). Without restriction it suffices to consider the initial condition $y_{0}(0)=\kappa$ and $y_{\alpha}(0)=0, \alpha \neq 0$ for some (small) deviation $\kappa$. To simplify the notation, in the sequel we write the spring constants as $m_{\alpha}=D e^{-\xi \alpha}$, with the shorthand

$$
D=\frac{B \varepsilon^{2} j_{X^{*}}}{1-e^{-\varepsilon L}}, \quad \xi=\frac{\varepsilon L}{N}
$$

To better understand the dynamics, let us consider the compound Poisson process $\mathcal{X}$ on $\mathbb{N}=\{0,1,2, \ldots\}$ starting from $\mathcal{X}(0)=0$, with the rate for the transition $\alpha \rightarrow \alpha+\gamma$ equal to $k(\alpha, \alpha+\gamma)=m_{\gamma} / \Gamma$ for all $\alpha=0, \ldots, N-1(\bmod N), \gamma=1, \ldots, N-1$, and zero otherwise. The time-dependent probabilities $p_{\alpha}(t)=\boldsymbol{P}[\mathcal{X}(t)=\alpha]$ satisfy the Master equation (with $p_{\alpha} \equiv 0$ whenever $\alpha<0$ )

$$
\begin{aligned}
\dot{p}_{\alpha} & =\sum_{\gamma>0}\left[p_{\alpha-\gamma} k(\alpha-\gamma, \gamma)-p_{\alpha} k(\alpha, \alpha+\gamma)\right] \\
& =\frac{1}{\Gamma} \sum_{\gamma>0} m_{\gamma}\left[p_{\alpha-\gamma}-p_{\alpha}\right]
\end{aligned}
$$


to be solved with the initial condition $p_{\alpha}(0)=\delta_{\alpha 0}$. By replacing $\alpha$ with $-\alpha$ it gets the form

$$
\dot{p}_{-\alpha}=\frac{1}{\Gamma} \sum_{\gamma>0} m_{\gamma}\left[p_{-(\alpha+\gamma)}-p_{-\alpha}\right], \quad \alpha=0,-1,-2, \ldots
$$

Comparing with (11) we observe that the $y_{\alpha}(t), \alpha=0,-1,-2, \ldots(\bmod N)$, solve a similar problem on the ring (now running in the opposite direction). More precisely, we have exactly that

$$
\begin{aligned}
y_{-\alpha}(t) & =\kappa \sum_{\alpha=0}^{+\infty} p_{\alpha+k N}(t), \quad \alpha=0,1, \ldots, N-1 \\
& =\kappa \boldsymbol{P}[\mathcal{X}(t)=\alpha \bmod N]
\end{aligned}
$$

We can thus obtain the solution to 11 via solving the compound Poisson process $\mathcal{X}(t)$, which is well-known - see e.g. [5]. The latter is characterized by a Poisson counter with intensity

$$
\Lambda=\frac{1}{\Gamma} \sum_{\alpha>0} m_{\alpha}=\frac{D}{\Gamma} \frac{1}{e^{\xi}-1}
$$

and by geometrically distributed jumps $\mathcal{J}$, with probabilities $\boldsymbol{P}[\mathcal{J}=\alpha]=\left(e^{\xi}-1\right) e^{-\xi \alpha}$ and with the first two moments

$$
\langle\mathcal{J}\rangle=\frac{1}{1-e^{-\xi}}, \quad\left\langle\mathcal{J}^{2}\right\rangle=\frac{1+e^{-\xi}}{\left(1-e^{-\xi}\right)^{2}}
$$

Then, by applying standard results on Poisson processes, [5], we immediately obtain

$$
\begin{aligned}
p_{0}(t) & =e^{-\Lambda t}=e^{-\frac{D t}{e^{\xi}-1}} \\
\langle\mathcal{X}(t)\rangle & =\langle\mathcal{J}\rangle \Lambda t=\frac{e^{\xi}}{\left(e^{\xi}-1\right)^{2}} \frac{D t}{\Gamma} \\
\operatorname{Var} \mathcal{X}(t) & =\left\langle\mathcal{J}^{2}\right\rangle \Lambda t=\frac{e^{\xi}\left(e^{\xi}+1\right)}{\left(e^{\xi}-1\right)^{3}} \frac{D t}{\Gamma}
\end{aligned}
$$

In words, the process $\mathcal{X}(t)$ leaves the origin and on average moves with speed $\langle\mathcal{J}\rangle \Lambda$ while diffusing. Therefore by the correspondence (13), the initial perturbation $y$ located at the first probe starts to decay exponentially fast and moves in the negative direction (with respect to the drift). It reaches again the origin in a somewhat delocalized form, and so on until it spreads over the whole circle. This way we detect three a priori different times scales:

(I) the characteristic time of initial decay of the perturbation at the origin,

$$
\tau_{0}=\frac{1}{\Lambda}=\frac{\Gamma}{D}\left(e^{\xi}-1\right)
$$


(II) the period of the echo effect after which the Poisson wave visits the origin,

$$
\tau_{\text {echo }}=\frac{N}{\langle\mathcal{J}\rangle \Lambda}=\frac{\left(e^{\xi}-1\right)^{2}}{e^{\xi}} \frac{\Gamma N}{D}
$$

(III) the relaxation time defined as a characteristic time in which the wave spreads over the whole circle,

$$
\tau_{\text {relax }}=\frac{N^{2}}{\left\langle\mathcal{J}^{2}\right\rangle \Lambda}=\frac{\left(e^{\xi}-1\right)^{3}}{e^{\xi}\left(e^{\xi}+1\right)} \frac{\Gamma N^{2}}{D}
$$

In particular, observing that

$$
\frac{\tau_{\text {echo }}}{\tau_{0}}=\left(1-e^{-\xi}\right) N, \quad \frac{\tau_{\text {relax }}}{\tau_{\text {echo }}}=\frac{e^{\xi}-1}{e^{\xi}+1} N
$$

we see that all three time scales are well separated on the assumption $\varepsilon L=\xi N \gg 1$ and $N \gg 1$ : Whenever $N \gg 1$ we always have

$$
\tau_{0} \ll \tau_{\text {echo }} \ll \tau_{\text {relax }}
$$

Altogether they complete the picture of a surprisingly rich relaxation mechanism which maintains the (moving) crystal pattern dynamically stable with respect to local perturbations.

\section{CONCLUSION}

The relaxation towards nonequilibrium conditions will in general not follow gradient flow in which a free energy is followed in its steepest descent. That remains true for the return to spatial patterns that are stabilized via driving. As a simple scenario we have analyzed the dynamical stabilization of the (moving) crystal pattern formed by equidistantly distributed heavy probes on the ring and locally interacting with a nonequilibrium bath. We have shown that the relaxation of small perturbations takes the form of a compound Poisson process and we have identified several relevant time scales.

Acknowledgments: KN acknowledges the support from the Grant Agency of the Czech Republic, grant no. 17-06716S.

[1] U. Basu, P. de Buyl, C. Maes, and K. Netočný, Driving-induced stability with long-range effects. EPL 115, 30007 (2016). 
[2] C. Boldrighini, R.L. Dobrushin and Yu.M. Sukhov, One-dimensional hard rod caricature of hydrodynamics. J. Stat. Phys. 31, 577-616 (1983).

[3] T. Demaerel, C. Maes and K. Netočný, Stabilization in the Eye of a Cyclone. Annales Henri Poincarè 19, 2673-2699 (2018).

[4] R.L. Dobrushin and J. Fritz, Non-equilibrium dynamics of one-dimensional infinite particle systems with a hard-core interaction. Comm. Math. Phys. 55, 275-292 (1977).

-, Non-equilibrium dynamics of two-dimensional infinite particle systems with a singular interaction. Comm. Math. Phys. 57, 67-81 (1977).

[5] J. F. C. Kingman, Poisson Processes, Oxford Studies in Probability, 1st Edition (Clarendon Press, 1993).

[6] H. K. Khalil, Nonlinear systems (Prentice Hall, 2002).

[7] C. Maes and K. Netočný, Non-reactive forces and pattern formation induced by a nonequilibrium medium. https://arxiv.org/abs/1711.05168

[8] C. Maes, K. Netočný and B. Wijnants, Steady state statistics of driven diffusions., Physica A 387, 2675-2689 (2008).

[9] On Dobrushin's Way. From Probability Theory to Statistical Physics. Eds. R.A. Minlos, Senya Shlosman and Yu. M. Suhov. American Mathematical Society Translations - Series 2 Advances in the Mathematical Sciences 